\title{
Executive Function and School Adjustment in Upper Elementary School Students: Mediating Effect of Self-Efficacy
}

\author{
Jae Eun Jeong ${ }^{1}$, Nana Shin ${ }^{2}$ \\ M. A., Department of Child Development \& Intervention, Ewha Womans University, Seoul, Korea ${ }^{1}$ \\ Assistant Professor, Department of Child Development \& Intervention, Ewha Womans University, Seoul, Korea ${ }^{2}$ \\ 학령 후기 아동의 실행기능과 학교적응: 자기효능감의 매개효과 \\ 정재은 ${ }^{1}$, 신나나 ${ }^{2}$ \\ 이화여자대학교 아동학과 석사 ${ }^{1}$, 이화여자대학교 아동학과 조교수 ${ }^{2}$
}

Objectives: The main purpose of this study was to examine the mediating effect of self-efficacy on the relationship between executive function (i.e., inhibition, working memory, and shifting) and school adjustment in upper elementary school students.

Methods: A total of 69 upper elementary school students participated in this study. They responded to questions regarding their perceived self-efficacy and school adjustment, and in addition, three tasks of executive function were administered: Stroop color and word test, digit span test, and trail making test.

Results: The subjects' perceived self-efficacy was found to partially mediate the relationship between their ability to inhibit and shift and school adjustment. However, the relationship between working memory and school adjustment was not mediated by self-efficacy.

Conclusion: This study highlights the importance of executive function and self-efficacy on school adjustment during the upper elementary school period. Findings from this study may be useful for the development of a self-efficacy enhancement program for upper elementary school students.

Keywords: executive function, self-efficacy, school adjustment

\section{Introduction}

인간은 성장하면서 다양한 환경에 접하게 되고, 이러한 환경 에 성공적으로 적응하기도 실패하기도 한다. 다양한 환경 중 개인과 학교 환경 간에 조화로운 관계를 형성하는 과정을 학 교적응이라고 하는데, 초기에는 주로 학업성취를 중심으로 정 의되었지만, 점차 아동의 사회정서적인 측면과 행동적인 측 면을 포함하는 개념으로 변화하였다(Birch \& Ladd, 1997). 이 러한 관점에서 학교적응은 아동이 성공적으로 학업 성취를

Corresponding Author: Nana Shin, Assistant Professor, Department of Child Development \& Intervention, Ewha Womans University, 52 Ewhayeodae-gil, Seodaemun-gu, Human Ecology Building 402-1, 03760, Seoul, Korea

E-mail: nanashin@ewha.ac.kr
이루는 것뿐만 아니라, 학교 환경에 관심을 가지고 참여하고 (Ladd, Kochenderfer, \& Coleman, 1997), 학교 내 대인관계나 규 칙, 그리고 질서 등을 따르는 데 있어 적절하고 조화로운 행동 을 하며, 그 안에서 편안함과 만족감을 경험하는 것을 의미한 다(Min, 1994). 초등학교 시기는 다양한 지식 습득, 또래와의 원만한 관계 형성, 적절한 성취동기를 지향하는 것이 발달 과 제(Lynch \& Cicchetti, 1997)이며, 환경과의 상호작용을 통해 개인의 성격과 행동특성이 결정되는 시기(Rubin, Bukowski, \& Parker, 2006)라는 점을 고려해 볼 때 학교적응의 중요성이 더

(c)The Korean Association of Child Studies

This is an Open Access article distributed under the terms of the Creative Commons Attribution Non-Commercial License (http:// creativecommons.org/licenses/by-nc/4.0) which permits unrestricted noncommercial use, distribution, and reproduction in any medium, provided the original work is properly cited. 
욱 강조되는 시기이다. 특히 우리나라 아동들은 학교에서 보 내는 시간이 상대적으로 길고, 중기 청소년기까지 학교 이외 의 다른 대안적인 진로기제가 부족한 상황에 놓여있기 때문에 학교적응의 문제를 더욱 중요하게 다루어야 할 필요가 있다.

학령기 학교적응의 중요성을 반영하듯, 학령기 아동을 대 상으로 학교적응이 발달에 미치는 영향을 탐색하는 연구들이 많이 수행되고 있다. 이러한 연구들에서는 이 시기 학교 부적 응이 학업중단의 주요 원인이며(Ministry of Education, 2018), 우울(Y. S. Cho \& Lee, 2010; Park \& Park, 2016), 일상적 스트레 스(Jang, Choi, \& Lee, 2016), 외현화 문제행동(Aunola, Stattin, $\&$ Nurmi, 2000) 등과 같은 부정적 발달 결과와 낮은 학업성취 도(M.-S. Lee \& Lee, 2009) 등과 관련됨을 보고하고 있다. 이러 한 문제를 경험하는 아동의 수는 중학교와 고등학교에 진학하 면서 더욱 증가하는데, 이는 개입뿐 아니라 예방적 측면에서 학교적응에 대한 연구가 초등학생을 대상으로 이루어질 필요 가 있음을 시사한다. 따라서 본 연구에서는 학령기 아동의 학 교적응에 영향을 미치는 아동의 개인적 자원을 탐색함으로써, 학교 부적응 문제를 예방하고, 개입하기 위한 방안을 제안하 고자 하였다.

아동이 가지고 있는 다양한 개인적 자원들 중에서 다양한 상황적 변화와 도전을 경험할 때 중요한 역할을 담당하는 실 행기능(Luna \& Sweeney, 2004)은 학교적응을 예측하는 주요 변인 중 하나로 제안된다. 실행기능은 사고, 정서, 그리고 행 동을 관리하고 통제하는 의식적인 고등 인지기능을 의미하며 (Zelazo \& Müller, 2002), 적응적인 반응을 위해서는 필요한 정 보에 주의를 기울이고, 불필요한 정보에 대한 반응은 억제하 며, 다양한 사고를 유연하게 할 수 있어야 한다는 점(Zelazo et al., 2003)에서 아동의 학교적응과 밀접한 관련이 있다. 실행 기능은 하나의 단일요인이 아닌 다차원적 요인으로 구성되 는데(Diamond, 2013; Pessoa, 2009), 실행기능을 구성하고 있 는 요인들에 대한 의견은 연구자들에 따라 다소 차이를 보인 다. 하지만 대부분의 연구에서 억제 능력(inhibition), 작업 기 억(working memory), 그리고 전환 능력(shifting)을 핵심 요인으 로 강조하고 있다. 실행기능은 이를 관장하는 전두엽 기능이 활성화되기 시작하는 유아기에 급격하게 발달하지만(Carlson, 2005), 각 구성요소들의 발달 속도는 다소 차이를 보인다 (Welsh, 2002). 즉, 억제 능력은 초기 청소년기까지(Williams, Ponesse, Schachar, Logan, \& Tannock, 1999), 작업 기억은 청소 년기까지(Beveridge, Jarrold, \& Pettit, 2002), 전환 능력은 후기 아동기까지(Cepeda, Kramer, \& Gonzalez de Sather, 2001) 지속 적으로 발달하는 것으로 보고되고 있다. 초등학교 고학년 시
기를 대상으로 한 연구들에서는 이 시기 실행기능이 지속적으 로 발달하기는 하지만, 그 발달 속도는 다소 완만해지는 것으 로 보고하고 있다(Anderson, 2002). 국내 초등학생을 대상으로 실시된 연구(M.-J. Lee \& Hong, 2006)에서도 저학년 시기 동 안, 그리고 저학년에서 고학년으로의 전환기에 실행기능의 각 영역에 발달적 변화가 관찰되었지만, 고학년 시기 동안에는 유의한 차이가 없음을 보고하였다. 그럼에도 불구하고 대부분 의 실행기능 연구들이 영유아기를 대상으로 실시되어 왔기 때 문에 학령기 아동을 대상으로 실행기능을 살펴보는 연구가 보 다 활성화될 필요가 있으며, 학령기 아동의 실행기능이 다양 한 발달영역에 미치는 영향이 탐색될 필요가 있다.

아동의 실행기능이 학교적응에 미치는 영향을 구체적으로 살펴보면, 먼저 개인의 필요에 따라 자신의 인지와 감정을 적 절하게 통제할 수 있는 억제 능력(Diamond, 2013)이 높을수 록 아동의 학업 태도가 긍정적이었으며, 교실 내에서 문제가 될 만한 행동들을 더 적게 보이는 것으로 보고되었다(Monette, Bigras, \& Guay, 2011; Riggs, Blair, \& Greenberg, 2004). 또한 새 로운 정보를 일시적으로 기억하여 정보를 처리하는 능력인 작 업 기억(Baddeley, 2003)이 더 높은 아동의 경우 높은 수준의 학 업 성취를 보였다(Monette et al., 2011). 그리고 두 가지 이상의 서로 다른 개념들을 자신의 목표에 따라 적절하게 적용할 수 있는 전환 능력(Scott, 1962)이 높은 아동일수록 학업적으로 더 유능하였으며, 또래가 평가한 친사회적 행동 점수가 더 높 았다(Zorza, Marino, \& Mesas, 2016). 이러한 선행연구들을 고 려해볼 때, 아동의 실행기능이 높을수록 학교에서 적응을 더 잘하지만, 실행기능의 하위요인에 따라 학교적응에 미치는 영 향도 다소 차이가 있을 것으로 가정해 볼 수 있다.

한편, 아동의 개인적 능력과 학교적응 간의 관계가 자기효 능감에 의해 매개되고 있음이 여러 선행연구들에 의해 보고 되고 있는데(Jung, 2008; Kang \& Kim, 2014; H. N. Lee \& Lee, 2012), 실행기능이 아동의 개인적 능력의 한 부분이라는 점에 서 이를 실행기능과 자기효능감, 그리고 학교적응 간의 관계 에도 적용해 볼 수 있다. 자기효능감은 미래의 상황을 다루기 위해 요구되는 행동들을 자신이 얼마나 잘 이행할 수 있는지 에 대한 개인적 믿음을 의미하는 것으로(Bandura, 1982), 아동 이 자신이 앞으로 수행할 행동이 성공적일 것이라는 확신을 가지게 함으로써 결과적으로 특정 행동을 수행하게 만드는 데 중요한 요소로 기능한다(Bandura, 1977). 이러한 측면에서 여 러 선행 연구들은 자기효능감과 학교적응 간 관계를 살펴보았 으며, 자기효능감이 높을수록 아동이 학교에서 보다 성공적으 로 기능하고 적응한다는 것을 밝힌 바 있다(Coleman, 2003; H. 
N. Lee \& Lee, 2012; J.-I. Lee, Kim, \& Han, 2014). 이를 구체적 으로 살펴보면, 아동의 자기효능감이 높을수록 학업태도가 좋 고(Y.-J. Cho, 2005), 학업에 대한 동기와 학업성취도가 높았으 며(Bandura, 1993; Schunk, 1989), 교사와 친구들과 긍정적 관 계를 형성하였다(Liem, Lau, \& Nie, 2008). 즉, 아동이 스스로 의 능력에 대해 믿음을 가지고 있을수록 학교 상황에서 요구 되는 관계적, 학업적 측면에 더 잘 적응하는 것을 알 수 있다.

자기효능감은 실행기능과도 관련되는데, 개인의 실행기 능 수준이 높을수록 주어진 과제들을 성공적으로 수행할 가능 성이 높아지며(LeFevre et al., 2013; Visu-Petra, Cheie, Benga, \& Miclea, 2011), 이는 곧 높은 자기효능감을 형성하도록 도울 것 이라 예측해 볼 수 있다. 실제로 실행기능에서의 결함은 낮은 학업적 자기효능감과 관련이 있었으며(Gambin \& Święcicka, 2015), 초등학교 1학년 시기에 실행기능의 구성 요소인 억제 통제를 포함한 개념인 적응적/의도적 통제가 높을수록 2학년 시기에 학업적 자아효능감이 높은 것으로 보고되었다(Liew, McTigue, Barrois, \& Hughes, 2008). 또한 실행기능과 관련된 상 위인지 전략을 더 잘 사용하고(Chung \& Kim, 2012), 스스로를 더 잘 통제할수록(Ju, 2011), 아동이 더 높은 자기효능감을 보 고하였다. 이외에도 실행기능과 스스로의 능력에 대한 개인적 인 느낌이나 가치감을 의미하는 자기유능감(Harter, 1982) 간 의 관계를 살펴본 연구도 보고되었는데, 청소년의 실행기능이 높을수록 자기유능감이 높은 것으로 나타났다(Y. Lee \& Kim, 2018). 이러한 연구들에 근거해 볼 때, 스스로의 주의, 사고, 행 동을 관리하고 통제하는 능력이 높을수록 학교 환경에서 주어 진 과제들을 성공적으로 수행함으로써 높은 자기효능감을 형 성할 것으로 예측해 볼 수 있다.

지금까지 살펴본 것처럼 아동의 실행기능, 자기효능감과 학교적응은 서로 관련이 있으며, 아동의 실행기능이 학교적응 에 미치는 영향은 자기효능감에 의해 매개될 것으로 가정해 볼 수 있다. 이는 Bandura (1977)가 제안한 '자기조절체계(selfregulatory system)'를 통해서도 설명될 수 있는데, 인간은 자기 조절체계를 가지고 있기 때문에 개인의 행동, 환경 속에서 일 어나는 사건, 그리고 개인의 인지적 요인 간의 상호작용을 통 해 능동적으로 조절되는 존재라 하였다. 이 때 개인의 인지적 요인이 실제 수행으로 이어지기 위해서는 자기효능감의 역할 이 중요하다고 강조하였다. 비록 이러한 가정을 실제로 확인 한 연구는 나타나고 있지 않지만, 스스로의 능력에 대한 느낌 이나 가치감을 의미하는 자기유능감이 실행기능이 학교생활 적응에 미치는 영향을 매개하는 것으로 보고되었으며(Y. Lee $\& \mathrm{Kim}, 2018)$, 여러 선행연구들을 통해 아동의 정서지능(Kang
\& Kim, 2014), 정서조절능력(H. N. Lee \& Lee, 2012), 의사소 통수준(Jung, 2008) 등과 같이 아동이 지니고 있는 다양한 자 원들이 학교적응에 미치는 영향을 자기효능감이 매개하는 것 으로 확인되었다. 이러한 선행연구들에 근거하여, 본 연구에 서는 초등학교 고학년 아동을 대상으로 개인내적 자원인 실 행기능의 세 가지 구성요소(i.e., 억제 능력, 작업 기억, 전환 능 력)가 학교적응에 미치는 영향이 자신의 능력에 대한 믿음을 의미하는 자기효능감에 의해 매개되는지 살펴보았다. 자기효 능감이 앞서 제시한 자기유능감보다 인지적인 측면이 더 강조 되는 개념이라는 점을 고려해 볼 때, 고등 인지기능을 뜻하는 실행기능과 보다 밀접하게 관련될 것으로 가정하였다. 현재까 지 수행된 실행기능 연구들이 대부분 영유아를 대상으로 수행 된 반면, 본 연구는 학령기를 대상으로 과제 수행을 통해 실행 기능을 측정함으로써 실행기능 연구에 다양한 발달단계를 포 함하는 데 기여하고자 하였다. 본 연구의 연구문제는 다음과 같다.

\section{연구문제 1}

학령 후기 아동의 실행기능이 학교적응에 미치는 영향을 자 기효능감이 매개하는가?

1-1. 학령 후기 아동의 억제 능력이 학교적응에 미치는 영 향을 자기효능감이 매개하는가?

1-2. 학령 후기 아동의 작업 기억이 학교적응에 미치는 영 향을 자기효능감이 매개하는가?

1-3. 학령 후기 아동의 전환 능력이 학교적응에 미치는 영 향을 자기효능감이 매개하는가?

\section{Methods}

\section{연구대상}

본 연구의 대상은 초등학교 4, 5, 6학년에 재학 중인 아동 69 명 이다. 본 연구의 대상을 초등학교 고학년으로 선정한 이유는 이 시기 실행기능에 문제가 있을 경우 학교생활 부적응을 경 험할 수 있으며(Y. H. Shin, Yun, \& Lee, 2010), 이는 청소년기 에 이르기까지 심리사회적 적응에 상당히 영향을 미칠 수 있 기 때문이다(DuBois, Eitel, \& Felner, 1994). 연구대상의 사회인 
구학적 특성을 살펴보면, 성별의 경우 남아가 42 명 $(61 \%)$, 여 아가 27 명(39\%)으로 남아의 비율이 여아보다 높았다. 연령은 만 10세 14명(20\%), 만 11세 25명(36\%), 만 12세 30명(44\%)이 었으며, 학년은 4학년 18명(26\%), 5학년 25명(36\%), 6학년 26 명 $(38 \%)$ 이었다.

\section{측정도구}

본 연구의 조사도구는 실행기능을 측정하기 위한 세 가지 과 제와 자기효능감과 학교적응을 측정하기 위한 질문지로 구성 되어 있다. 실행기능은 과제를 제시하고 이에 대한 아동의 수 행을 연구자가 직접 측정하였으며, 자기효능감과 학교적응은 질문지 문항에 아동이 직접 응답하도록 하였으며, 필요한 경 우 연구자가 문항에 대한 설명을 제시하였다.

\section{실행기능}

실행기능은 억제 능력, 작업 기억, 전환 능력의 세 가지를 측정 하였으며, 이 중 억제 능력은 스트룹 아동 색상-단어 검사, 작 업 기억은 한국판 아동용 웩슬러 지능검사 4판 중 숫자 따라 하기 과제, 전환 능력은 아동 색 선로검사를 사용하여 측정하 였다. 실행기능은 서로 다른 기능을 담당하는 다차원적 변인 으로 구성되어 있으며(Zelazo \& Müller, 2002), 점수를 산출하 는 방법도 각기 다르기 때문에 본 연구에서는 평균을 산출하 지 않고 각 하위요인을 분석에 사용하였다.

억제 능력 억제 능력은 Golden (1978)이 개발하고 M. Shin과 Park (2006)이 한국판으로 표준화한 스트룹 아동 색상-단어 검 사(Stroop Color and Word Test [SCWT])를 사용하여 측정하였 다. 검사는 총 세 페이지(단어, 색상, 색상-단어)로 이루어져 있 으며, 각 페이지마다 색상명 또는 문자로 'XXXX'표시가 20개 씩 5열로 총 100 개가 제시되었다. 단어 페이지의 경우 '파랑,' ‘빨강,' ‘초록'이라는 글자가 검은 색으로 인쇄되어 있으며, 색 상 페이지의 경우 'XXXX'표시가 파란색, 빨간색, 초록색으로 인쇄되어 있다. 마지막으로 색상-단어 페이지의 경우 색상을 의미하는 글자가 의미하는 바와는 다른 색상으로 인쇄되어 있 다(e.g., 초록이라는 글자가 빨간색으로 인쇄되어 있음). 아동 에게 한 번에 한 페이지씩 보여주었으며, 제한된 45초 동안 단 어 페이지의 경우 제시된 단어를 얼마나 정확하고 빠르게 읽 는지, 색상 페이지의 경우 제시된 색상을 얼마나 정확하고 빠 르게 명명하는지, 마지막으로 색상-단어 페이지의 경우 주어
진 글자 자극을 무시하고 얼마나 정확하고 빠르게 색상을 명 명하는지를 확인하였다. 45 초 동안 총 몇 개의 색상명 또는 'XXXX'표시를 각 페이지의 지시사항에 따라 성공적으로 명명 하였는지를 측정하였으며, 아동이 잘못 명명하였을 경우 손 가락으로 틀린 부분을 가리켜 다시 명명하도록 하였다. 아동 이 각 페이지에서 첫 시도에서 명명에 성공한 개수를 저작권 자로부터 구매한 프로그램에 입력하였고, 프로그램을 통해 기 존에 축적된 동 연령 집단이 획득한 점수 데이터들과 비교하 여 표준화된 T점수가 산출되었다. 본 연구에서는 우세한 자극 인 글자 자극을 무시하고 상대적으로 덜 우세한 자극인 색깔 자극을 명명하여 얻은 색상-단어 페이지의 $\mathrm{T}$ 점수를 분석 자료 로 사용하였다. 단어 페이지의 T점수는 아동이 기본적으로 읽 기 능력에 문제가 있는지, 색상 페이지의 T점수는 아동이 색 상 인지에 문제가 있는지, 또는 글자 자극보다 색깔 자극이 더 우세한 자극인지를 확인하기 위한 목적으로 사용되었기 때문 에 억제 능력의 점수에는 포함하지 않았다. 단어 검사와 색상 검사에서 기본적인 읽기 능력과 색상 인지에 문제가 있는 아 동은 없었다.

작업 기억 작업 기억을 측정하기 위해 한국판 아동용 웩슬러 지능검사 4판(Korean Wechsler Intelligence Scale for Children IV [K-WISC IV]; Kwak, Oh, \& Kim, 2011)의 소검사인 '숫자 바로 따라 하기(digit span forward)'와 '숫자 거꾸로 따라 하기(digit span backward)'를 사용하였다. 두 가지 검사 모두 두 자리부터 시작하여 한 자리씩 늘어나며, 같은 자리의 숫자를 총 2 회씩 (시행1, 시행2) 시행하며, 최대 여덟 자리까지 제시된다. 시행 한 개를 통과할 때마다 1점씩 부여하고, 같은 자리의 숫자의 두 가지 시행을 모두 틀릴 경우 검사를 중단한다. 또한 각 시행 에서 제시되는 숫자는 한 번씩만 아동에게 들려주며, 아동이 다시 들려주기를 요청할 경우, 생각나는 대로 편하게 숫자를 말해주면 된다고 대답하였다.

먼저 연구자가 불러준 숫자를 그대로 따라하는 숫자 바로 따라 하기를 진행하였고, "선생님이 숫자를 말하고 난 후에 그 대로 따라 말해주면 돼요.”라고 설명 후 시작하였다. 숫자 바 로 따라 하기 과제는 정보의 유지만을 요하는 단기기억 과제 (H. Kim \& Park, 2003)이지만, 아동의 작업 기억을 평가함에 앞서 단기기억, 일반지능, 청각적, 그리고 언어적 문제 등이 있 는지 확인하기 위해 실시하였으며, 이 과제를 수행하는 데 문 제가 있는 아동은 없었다. 숫자 바로 따라 하기를 마친 후 연구 자가 불러준 숫자를 역순으로 재배열하여 말하는 숫자 거꾸로 따라 하기 과제를 시행하였다. "이번에는 선생님이 숫자를 다 
말하고 난 후에 그 숫자들을 거꾸로 말해주면 돼요. 만약 선생 님이 8-2라고 하면 뭐라고 말해야 하죠?”라고 한 뒤, 아동이 수 행 방법을 이해했는지 확인 후 시작하였다. 본 연구에서는 숫 자 거꾸로 따라 하기 과제에서 아동이 획득한 점수를 사용하 였으며, 총 14 번의 시행이 실시되므로 아동이 획득할 수 있는 점수의 범위는 0-14점이다.

전환 능력 전환 능력은 Reitan (1979)의 선로 잇기 검사(Trail Making Test [TMT])를 문화 및 언어의 영향을 최소화하기 위 하여 Williams 등(1995)이 수정 및 보완하여 개발한 아동 색 선 로 검사(Children's Color Trails Test [CCTT])를 M. Shin과 Koo (2006)가 한국판으로 표준화한 것을 사용하여 측정하였다. 숫 자가 적힌 원을 순서대로 연결해야 하는 CCTT-1과 숫자가 적 힌 원을 순서대로 연결하는 동시에 원의 색깔(분홍색 또는 노 란색)의 순서도 번갈아 가며 연결(e.g., 분홍색 $1 \rightarrow$ 노란색 2 $\rightarrow$ 분홍색 3)해야 하는 CCTT-2로 구성되어 있다. CCTT-1은 주의력 변경 및 지속적 시각 주의력(alternating and sustained visual attention)을 요구하며, 이에 더하여 CCTT-2는 상징 (symbol) 혹은 개념(concept)들(e.g., 숫자나 글자) 사이를 번갈 아 가며 각각의 순서를 따라가면서 범주를 계속해서 바꾸어 가는 능력을 포함한다. CCTT-1과 CCTT-2 과제를 각각 완성 하는 데 소요되는 시간과 과제 수행 도중 발생하는 오류 수를 측정하였으며, 측정 결과를 저작권자로부터 구매한 프로그램 에 입력하였다. 과제 완성 시간이 짧을수록 더 높은 점수가 부 여되며, 동 연령 집단이 획득한 점수 데이터들과 오류 수를 고 려하여 최종 표준화된 T점수가 산출되었다. 본 연구에서는 아 동의 CCTT-2 T점수를 최종 주의전환 점수로 사용하였으며, CCTT-1에서의 수행이 저하되고 CCTT-2에서의 수행이 양호 할 경우, 아동이 검사에 대한 불안을 느끼고 있음을 확인할 수 있는데(Heaton, Chelune, Talley, Kay, \& Curtiss, 1993), 본 연구 에서는 이러한 현상은 나타나지 않았다.

\section{자기효능감}

자기효능감을 측정하기 위하여 A. Y. Kim과 Cha (1996)가 개 발하고, A. Y. Kim (1997)이 수정한 일반적 자기효능감 척도를 $\mathrm{Han}$ (2002)이 초등학생에게 적합한 표현으로 수정한 것을 사 용하였다. 본 척도는 자신감, 자기조절 효능감, 그리고 과제난 이도 선호의 3 개 하위요인으로 구성되어 있다. 문항의 예로는 자신감(8문항)은 "나는 어려운 상황을 극복할 수 있는 능력이 있다.", 자기조절 효능감(11문항)은 “나는 일을 순서를 정해 차
례로 처리할 수 있다.”, 과제난이도 선호(5문항)는 “좀 실수를 하더라도 나는 어려운 일을 좋아한다.” 등이 있다. 각 문항은 5 점 Likert식 척도로 응답되도록 구성되어 있으며, 부정적 의미 에 해당하는 문항들은 역채점한 후 평균을 산출하였다. 따라 서 점수가 높을수록 자신감, 자기조절 효능감, 그리고 과제난 이도 선호가 높음을 의미하며, 각 하위요인들의 총합이 높을 수록 자기효능감이 더 높음을 의미한다. 세 하위요인들 간 상 관이 높고 $(r s=.50 \sim .58, p<.001)$, 탐색적 요인분석의 결과도 하나의 요인으로 수렴되어, 세 하위요인 간 평균을 산출하여 단일 요인으로 분석에 사용하였다. 전체 문항 간 내적합치도 (Cronbach's $\alpha$ )는 .89이었다.

\section{학교적응}

학교적응은 S. Cho (2014)가 학령 후기 아동을 대상으로 개발 하고 타당화한 학교적응 척도를 사용하여 측정하였다. 본 척 도는 학업태도/규칙준수, 또래와의 관계, 교사와의 관계, 그리 고 학교만족감의 4 개 하위요인으로 구성되어 있다. 문항의 예 로는 학업태도/규칙준수(9문항)는 "나는 수업시간에 집중하 여 듣는다.”, 또래와의 관계(7문항)는 “나는 학교에서 친구들 과 잘 어울린다.", 교사와의 관계(8문항)는 "담임선생님은 나 를 좋아하신다.", 학교만족감(6문항)은 “나는 학교생활에 만족 한다." 등이 있다. 각 문항은 4점 Likert식 척도로 구성되어 있 으며, 점수가 높을수록 학업태도/규칙준수, 또래와의 관계, 교 사와의 관계, 그리고 학교만족감 점수가 높음을 의미한다. 학 교적응의 네 하위요인 간 상관은 .43 .80 ( $p<.001)$ 이었으며, 탐색적 요인분석의 결과도 하나의 요인으로 수렴되었다. 따라 서 하위요인 간 평균을 산출하여 학교적응 전체 점수를 산출 하였으며, 문항 간 내적합치도(Cronbach's $\alpha$ )는 .95였다.

\section{조사절차 및 자료분석}

실행기능 과제 수행과 자기효능감과 학교적응에 관한 질문지 를 응답하는 데 걸리는 시간이 아동의 연령에 적합한지, 절차 는 적절한지, 그리고 과제의 난이도는 적합한지 등을 살펴보 기 위해 예비조사를 실시하였다. 예비조사는 4 학년 아동 2 명 과 6학년 아동 2명, 총 4명을 대상으로 실시하였다. 예비조사 결과, 학년에 관계없이 과제와 과제 제시 순서, 질문지 난이도 및 총 소요 시간이 적절한 것으로 판단되어 본 조사에서도 수 정 없이 그대로 사용하였다. 본조사는 2019년 1월부터 3월까 지 수행되었으며, 육아 관련 인터넷 사이트에 모집문건을 게 
시하거나 학원 방문 등의 방법으로 참여자를 모집하였다. 참 여 의사를 밝힌 아동과 부모님들에게 개별적으로 검사의 내용 과 목적, 절차에 대해 안내드리고 일정을 조정하였다. 검사는 참여자가 대학의 아동실험실을 방문하거나 혹은 연구자가 참 여자의 가정이나 학원 등을 방문하여 조용한 공간에서 실시하 는 방식으로 이루어졌으며, 최종 69명의 아동이 본 연구에 참 여하였다. 실행기능 검사에는 약 13 분 정도가 소요되었으며, 질문지 응답에는 약 10 분 정도가 소요되었다. 검사를 시작하 기 전, 아동에게 연구에 대해 설명하며 아동의 기본정보를 수 집하였다. 그 후, 아동과의 간단한 대화를 통해 친밀감을 형성 한 뒤 자기효능감과 학교적응 순으로 질문지를 작성하도록 요 청하였다. 질문지 작성이 완료되면 각 과제를 실시하기 전, 과 제 순서와 과제 수행 방법에 대해 설명한 후 간단한 연습 과제 시행 후 본 과제를 실시하였다. 과제는 작업 기억, 억제 능력, 전환 능력 과제 순으로 진행하였으며, 아동이 모든 과정을 마 친 후 참여에 대한 감사의 표시로 5,000원 상당의 문화상품권 을 제공하였다.

수집된 자료를 분석하기 위하여 SPSS 23.0 (IBM Co., Armonk, NY) 프로그램을 사용하였다. 먼저, 연구대상의 사 회인구학적 특성을 확인하기 위하여 빈도와 백분율을 확인 하였고, 조사도구의 문항 간 내적합치도를 살펴보기 위하여 Cronbach's의 $\alpha$ 값을 산출하였다. 다음으로, 연령에 따라 실행 기능의 각 구성요소에 차이가 있는지 살펴보기 위하여 일원분 산분석을 실시하였으며, 각 변인들 간의 상관관계를 알아보기 위해 Pearson의 적률상관계수를 산출하였다. 마지막으로, 실행 기능이 초등학생의 학교적응에 미치는 영향을 자기효능감이 매개하는지 살펴보기 위하여 Baron과 Kenny (1986)의 절차를 따라 3단계에 걸친 회귀분석을 실시하였다. 간접효과의 유의 성은 sobel test를 통해 검증하였다.

\section{Results}

\section{예비분석}

본 분석에 앞서 연령에 따라 실행기능이 차이를 보이는지 살 펴보기 위해 일원분산분석을 실시한 결과, 억제 능력, 작업 기 억, 전환 능력 모두에서 연령에 따른 차이는 유의하지 않았다. 따라서 추후 분석에서는 전체를 대상으로 분석하였다. 다음 으로 실행기능, 자기효능감 및 학교적응 간의 관계를 살펴보 기 위해 Pearson의 적률상관계수를 산출한 결과, 대부분의 변
인 간 상관이 통계적으로 유의하였다(Table 1). 이를 구체적으 로 살펴보면, 첫째, 실행기능과 학교적응의 경우 억제 능력 $(r$ $=.37, p<.01)$ 과 전환 능력 $(r=.45, p<.001)$ 은 학교적응과 정 적인 상관이 있었으며, 작업 기억은 학교적응과 통계적으로 유의미한 상관을 보이지 않았다. 즉, 억제 능력과 전환 능력이 높을수록 높은 수준의 학교적응을 보였다. 둘째, 실행기능과 자기효능감 간의 관계는 유의한 정적 상관을 보여, 억제 능력 $(r=.27, p<.05)$, 작업 기억 $(r=.29, p<.05)$, 그리고 전환 능력 $(r=.47, p<.001)$ 이 높을수록 자기효능감이 높았다. 마지막으 로, 아동의 자기효능감과 학교적응 간 관계를 살펴본 결과, 자 기효능감이 높을수록 학교에 적응을 더 잘하였다 $(r=.53, p<$ $.001)$.

Table 1

Correlations Among Executive Function, Self-Efficacy, and School Adjustment

\begin{tabular}{|c|c|c|c|c|c|}
\hline & 1 & 2 & 3 & 4 & 5 \\
\hline 1. Inhibition & - & & & & \\
\hline $\begin{array}{l}\text { 2. Working } \\
\text { memory }\end{array}$ & $.38^{* * *}$ & - & & & \\
\hline 3. Shifting & $.46^{* * *}$ & $.42^{* * *}$ & - & & \\
\hline 4. Self-efficacy & $.27^{*}$ & $.29^{*}$ & $.47^{* * *}$ & 一 & \\
\hline $\begin{array}{l}\text { 5. School } \\
\text { adjustment }\end{array}$ & $.37^{* *}$ & .22 & $.45^{* * *}$ & $.53^{* * *}$ & - \\
\hline Range & $30-77$ & $4-12$ & $31-71$ & $2.01 \sim 4.78$ & $1.79 \sim 3.97$ \\
\hline$M$ & 49.61 & 7.62 & 56.54 & 3.43 & 3.25 \\
\hline$S D$ & 9.86 & 1.77 & 7.85 & 0.54 & 0.50 \\
\hline
\end{tabular}

Note. $N=69$.

${ }^{*} p<.05 .{ }^{* *} p<.01{ }^{* * *} p<.001$.

\section{실행기능이 학교적응에 미치는 영향에서 자기 효능감의 매개효과}

아동의 실행기능이 학교적응에 미치는 영향을 자기효능감이 매개하는지 살펴보기 위하여, Baron과 Kenny (1986)의 3단계 접근법에 근거하여 단순회귀분석과 중다회귀분석을 실시하 였다. 매개효과가 성립하기 위해서는 1단계에서 독립변인인 아동의 실행기능이 종속변인인 학교적응에 유의한 영향을 미 
Table 2

Self-Efficacy as a Mediator of the Relationship Between Inhibition and School Adjustment

\begin{tabular}{cllllllc}
\hline Step & & & $B$ & $S E$ & $\beta$ & $R^{2}$ & $F\left(d f_{1}, d f_{2}\right)$ \\
\hline 1 & Inhibition $\rightarrow$ School adjustment & .02 & .01 & $.37^{* *}$ & .14 & $10.66^{* *}(1,67)$ \\
2 & Inhibition $\rightarrow$ Self-efficacy & .02 & .01 & $.27^{*}$ & .07 & $5.06^{*}(1,67)$ \\
& Inhibition $\rightarrow$ School adjustment & .01 & .01 & $.25^{*}$ & .33 & $16.57^{* * *}(2,66)$ \\
& Self-efficacy $\rightarrow$ School adjustment & .43 & .10 & $.46^{* * *}$ & &
\end{tabular}

Note. $N=69$.

${ }^{*} p<.05{ }^{* *} p<.01 .{ }^{* * *} p<.001$.

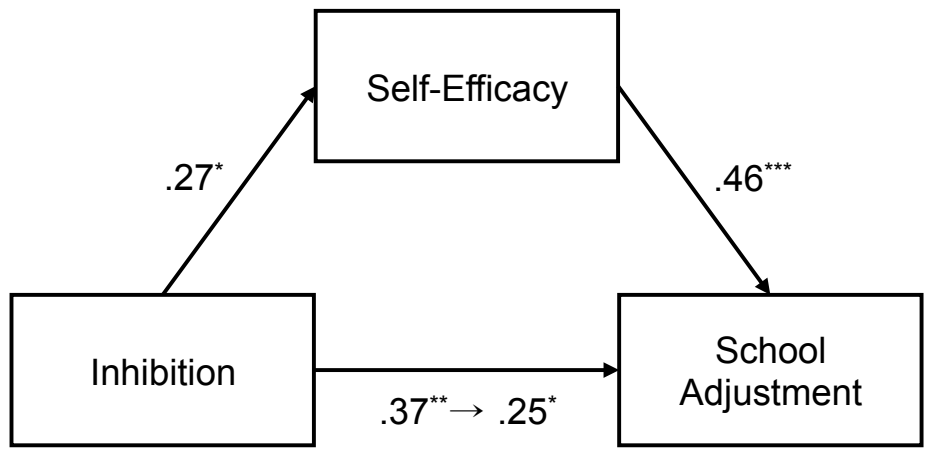

Figure 1. Self-efficacy as a mediator of the relationship between inhibition and school adjustment.

${ }^{*} p<.05 .{ }^{* *} p<.01 .{ }^{* * *} p<.001$.

쳐야 하고, 2 단계에서는 독립변인인 아동의 실행기능이 매개 변인인 아동의 자기효능감에 통계적으로 유의한 영향을 미쳐 야한다. 마지막으로 3 단계에서는 독립변인인 아동의 실행기 능과 매개변인인 아동의 자기효능감을 함께 투입하였을 때, 매개변인인 아동의 자기효능감이 종속변인인 학교적응에 통 계적으로 유의한 영향을 미쳐야 하며, 독립변인인 아동의 실 행기능은 종속변인인 학교적응에 더 이상 유의한 영향을 미치 지 않거나(완전매개), 회귀계수가 감소(부분매개)해야 한다. 매개효과의 통계적 유의성은 sobel test를 통해 검증하였다. 매 개효과의 전제조건을 충족하는지 확인해 본 결과, 작업 기억 의 경우 종속변인인 학교적응과 통계적으로 유의한 상관을 보 이지 않아 매개분석에서는 제외하였다. 억제 능력과 전환 능 력의 경우 자기효능감(억제 능력: $r=.27, p<.01$; 전환 능력: $r$ $=.47, p<.001$ )과 학교적응(억제 능력: $r=.37, p<.01$; 전환 능 력: $r=.45, p<.001)$ 과 모두 유의한 상관을 보여, 매개효과의 전제조건이 충족되었다.

먼저, 아동의 억제 능력이 학교적응에 미치는 영향에서 자 기효능감의 매개효과를 살펴본 결과(Table 2), 1 단계와 2 단계 에서 아동의 억제 능력은 종속변인인 학교적응 $(\beta=.37, p<$ $.01)$ 과 매개변인인 자기효능감 $(\beta=.27, p<.05)$ 에 유의한 영향 을 미쳤다. 마지막으로 3 단계에서 억제 능력과 자기효능감을
함께 투입한 결과, 자기효능감은 학교적응에 유의한 영향 $(\beta=$ $.46, p<.001)$ 을 미쳤으며, 억제 능력이 학교적응에 미치는 영 향은 1 단계에 비해 감소하였다 $\beta=.37, p<.01 \rightarrow \beta=.25, p<$ $.05)$. 즉, 아동의 자기효능감은 억제 능력과 학교적응 간의 관 계를 부분적으로 매개하였다(Figure 1). 매개효과의 유의성을 살펴보기 위해 sobel test를 실시한 결과, 아동의 억제 능력이 자기효능감을 통해 학교적응에 미치는 영향이 통계적으로 유 의하였다 $(z=4.72, p<.001)$.

다음으로, 아동의 전환 능력이 학교적응에 미치는 영향에 서 아동의 자기효능감의 매개효과를 살펴본 결과(Table 3), 1 단계와 2단계에서 아동의 전환 능력은 종속변인인 학교적응 $(\beta=.45, p<.001)$ 과 매개변인인 자기효능감 $(\beta=.47, p<.001)$ 에 유의한 영향을 미쳤다. 3 단계에서 전환 능력과 자기효능감 을 함께 투입한 결과, 자기효능감은 학교적응에 유의한 영향 $(\beta=.41, p<.001)$ 을 미쳤으며, 전환 능력이 학교적응에 미치 는 영향은 1 단계에 비해 감소하였다 $(\beta=.45, p<.001 \rightarrow \beta=$ $.26, p<.05)$. 즉, 아동의 자기효능감은 전환 능력과 학교적응 간의 관계를 부분적으로 매개하였다(Figure 2). 매개효과의 유 의성을 살펴보기 위해 sobel test를 실시한 결과, 아동의 억제 능력이 자기효능감을 통해 학교적응에 미치는 영향이 통계적 으로 유의하였다 $(z=3.86, p<.001)$. 
Table 3

Self-Efficacy as a Mediator of the Relationship Between Shifting and School Adjustment

\begin{tabular}{clllllll}
\hline Step & & & $B$ & $S E$ & $\beta$ & $R^{2}$ & $F\left(d f_{1}, d f_{2}\right)$ \\
\hline 1 & Shifting & $\rightarrow$ School adjustment & .03 & .01 & $.45^{* * *}$ & .20 & $16.61^{* * *}(1,67)$ \\
2 & Shifting & $\rightarrow$ Self-efficacy & .03 & .01 & $.47^{* * *}$ & .22 & $18.73^{* * *}(1,67)$ \\
3 & Shifting & $\rightarrow$ School adjustment & .02 & .01 & $.26^{*}$ & .33 & $16.10^{* * *}(2,66)$ \\
& Self-efficacy & $\rightarrow$ School adjustment & .38 & .11 & $.41^{* * *}$ & & \\
\hline
\end{tabular}

Note. $N=69$.

${ }^{*} p<.05 .{ }^{* * *} p<.001$.

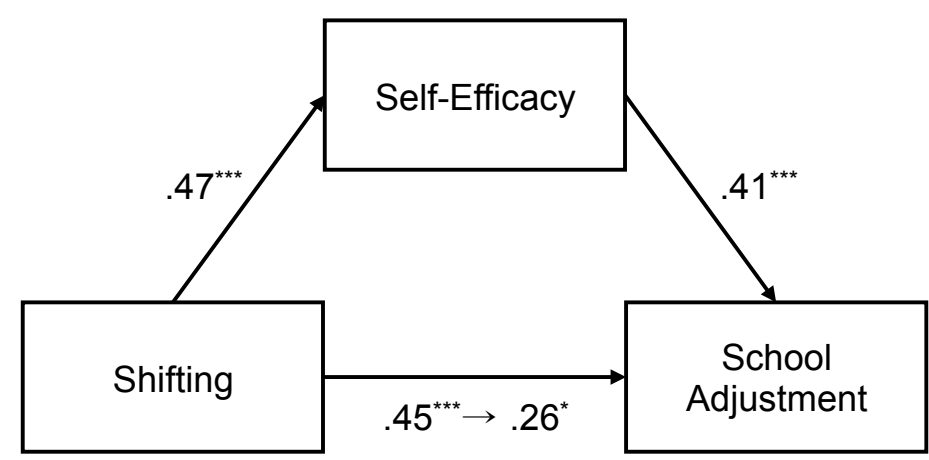

Figure 2. Self-efficacy as a mediator of the relationship between shifting and school adjustment.

${ }^{*} p<.05 .{ }^{* * *} p<.001$.

\section{Discussion}

본 연구는 초등학교 4-6학년생을 대상으로 아동의 실행기능 이 학교적응에 미치는 영향을 자기효능감이 매개하는지 탐색 하였다. 실행기능의 하위요인들인 억제 능력, 작업 기억, 전환 능력은 그 특성이 다르기 때문에(Zelazo \& Müller, 2002), 각각 을 분리하여 분석하였다. 본 연구의 주요 결과를 요약하고 논 의하면 다음과 같다.

아동의 실행기능이 학교적응에 미치는 영향을 자기효능감 이 매개하는지 살펴본 결과, 억제 능력과 전환 능력이 학교적 응에 미치는 영향은 자기효능감에 의해 부분 매개 되었다. 즉, 아동의 억제 능력과 전환 능력이 높을수록 자기효능감이 높았 으며, 그 결과 학교생활에 적응을 더 잘하였다. 실행기능이 학 교적응에 미치는 영향을 자기효능감이 매개하는지 살펴본 선 행연구가 없어 본 연구의 결과를 선행연구와 직접 비교하기 에는 어려움이 있다. 하지만 Bandura와 Cervone (1983)이 제안 한 것처럼 개인이 어떤 능력을 가지고 있는 것과 그 능력을 실 제로 사용하는 것은 구분되며, 그 사이를 매개하는 것이 자기 효능감이라는 점에 비추어 본 연구의 결과를 해석해 볼 수 있 다. 또한, 아동이 가지고 있는 자원이 행동에 영향을 미치는 과 정에서 자기효능감이 매개적 역할을 한 선행연구들을 통해서
도 본 연구의 결과를 해석해 볼 수 있다. 구체적으로, 선행연구 들은 초등학생의 정서지능(Kang \& Kim, 2014), 정서조절능력 (H. N. Lee \& Lee, 2012), 의사소통수준(Jung, 2008), 그리고 스 스로 지각한 사회적 지지(H.-S. Kim, 2019) 등이 자기효능감을 통해 학교적응에 영향을 미치는 것으로 보고하고 있다. 즉, 자 신의 능력에 대한 믿음이자, 목표를 설정하고 전략적으로 계 획한 후, 이를 실천으로 옮기도록 동기화시키는 역할을 하는 자기효능감(Bandura, 1982)이 아동이 학교에서 성공적으로 적 응할 수 있도록 도울 수 있는 심리적 자원이 될 수 있다는 것을 알 수 있다.

또한, 아동의 억제 능력과 전환 능력이 학교적응에 미치는 영향이 자기효능감에 의해 부분매개 되는 것으로 나타난 본 연구의 결과는, 아동이 자신의 욕구를 잘 통제하고, 유연성 있 는 사고를 지니는 것 자체도 학교 환경에 더 잘 적응하는 데 중 요한 특성으로 작용한다는 것을 시사한다. 이러한 연구결과는 실행기능의 억제 능력과 유사한 기질적 특성인 의도적 통제 (Rothbart, Sheese, \& Posner, 2007)가 높을수록 초등학생의 교 사와의 관계, 사회적 유능성 및 수업 참여도가 높은 것으로 나 타난 연구(Valiente, Lemery-Chalfant, Swanson, \& Reiser, 2008) 와 유사한 맥락에서 해석할 수 있다. 또한, 초등학생은 아니지 만, 중학생의 인지적 유연성이 높을수록 학교적응을 더 잘하 
는 것으로 보고된 연구(Do, 2015)와도 유사한 결과이다. 이러 한 결과를 통해 아동이 자신의 욕구를 잘 통제하고, 유연성 있 는 사고를 지니는 것이 학교 환경에 더 잘 적응하는 데 중요 한 특성임을 알 수 있다. 따라서 성공적인 학교적응을 위해서 는 아동의 억제 능력과 전환 능력을 최적의 상태로 발달시키 기 위해 아동의 자율성을 지지하고, 다양한 인지적 경험을 해 볼 수 있는 기회를 제공하는 것이 중요할 것이다. 정리하자면, 아동의 성공적인 학교적응을 위해서는 아동의 능력 증진과 아 동이 스스로 자신의 능력을 믿을 수 있도록 돕기 위한 개입의 필요성이 제기된다. 하지만 실행기능과 자기효능감을 모두 포 함하여 아동의 발달에 미치는 영향을 탐색한 연구가 나타나고 있지 않기 때문에, 이에 대한 다양한 연구가 이루어진다면 아 동의 인지 기술과 능력에 대한 믿음이 다양한 발달 영역에 미 치는 영향력의 차이를 파악할 수 있을 것이라 기대된다.

한편, 본 연구에서는 작업 기억이 학교적응에 미치는 영향 이 자기효능감에 의해 매개 되는지 여부는 살펴볼 수 없었는 데, 이는 독립변인인 작업 기억과 종속 변인인 학교적응 간의 상관이 유의하지 않았기 때문이다. 이러한 결과는 작업 기억 이 좋을수록 초등학생이 학교 수행에 더 적극적으로 참여하 고(Gathercole, Lamont, \& Alloway, 2006), 학교생활에 더 잘 적 응하는 것으로 나타난 결과(Sasser, Bierman, \& Heinrichs, 2015) 나, 본 연구대상의 연령과는 차이가 있지만 작업 기억이 중학 생의 학교에서의 유능성에 영향을 미쳤다는 결과(Jacobson, Williford, \& Pianta, 2011)와는 차이를 보인다. 하지만 초등학 생(Choi \& Lee, 2016)과 중학생의 작업 기억(M. Kim \& Ha, 2013)이 또래관계의 질과 유의미한 상관이 없는 것으로 나타 난 연구결과들과는 유사하다.

이처럼 작업 기억이 아동의 학교적응에 미치는 영향이 연 구마다 다르게 보고되는 이유는 두 가지 측면에서 해석할 수 있는데, 먼저, 학교적응에 포함되는 구성 요인이 연구마다 차 이를 보였기 때문일 수 있다. 즉, 학교적응은 크게 학업과 관련 된 측면과 대인관계로 구성되는데, 작업 기억이 이들 각각과 가지는 관계가 차이가 있기 때문으로 해석해 볼 수 있다. 본 연 구에서도 아동의 실행기능과 학교적응의 하위요인 간 상관을 추가로 살펴본 결과, 억제 능력과 전환 능력은 학교적응의 모 든 하위요인들과 정적인 상관이 있었던 반면, 작업 기억은 학 업태도/규칙준수 요인과만 유의한 상관을 보여 이러한 해석 을 뒷받침한다. 본 연구에서는 표본 수가 적어 학교적응을 전 체 점수를 산출하여 살펴보았지만, 추후 연구에서는 표본수를 확대하여 학교적응의 각 구성요소별로 실행기능과의 관련성 을 살펴본다면 작업 기업과 학교적응의 각 구성요소 간의 관
계를 더 명확히 살펴볼 수 있을 것이다. 또 다른 측면에서의 해 석은 작업 기억이 개념적으로 학교적응과 관련이 있지만, 본 연구의 표본 수가 적기 때문에 유의한 결과가 나오지 않았을 가능성이 있다. 실제로 우리나라 학생들의 경우 학업과 관련 된 요인이 학교적응에 미치는 영향이 매우 크다는 사실(M. S. $\mathrm{Kim}, 2009)$ 을 고려해 보았을 때, 학업과 관련된 요소에 영향 을 미치는 작업 기억(Daneman \& Carpenter, 1980; Engle, 2002; Jin, 2018)이 아동의 학교적응에도 상당한 영향을 미칠 것이라 유추해 볼 수 있다. 본 연구에서도 작업 기억과 학교적응 간 상 관이 유의하지는 않았지만 유의 수준에 근사한 것 $(p=.07)$ 으 로 나타나, 이러한 가능성을 제안하며 추후 연구에서는 보다 많은 아동들을 대상으로 연구를 진행하여 작업 기억과 학교적 응 간의 관계를 명확하게 규명할 필요가 있다.

종합하면, 본 연구에서는 스스로의 인지, 사고, 정서를 통제 하는 능력인 실행기능이 아동이 학교에서 성공적으로 적응하 는 데 중요한 역할을 담당하며, 이러한 과정에서 아동 스스로 가 자신의 능력에 대해 믿음을 가지는 것이 중요한 매개적 역 할을 담당함을 밝혔다. 특히, 실행기능의 하위 요소 중 우세한 반응을 억제하고 유연성 있는 사고를 할 수 있는 능력은 학교 적응과 관련이 있었지만, 새로운 정보를 일시적으로 기억하여 정보를 처리하는 능력은 학교적응과 관련이 없는 것으로 나 타나 학령 후기 아동의 학교적응에 영향을 미치는 인지 능력 에 대한 세부적인 접근이 필요함을 제안하였다. 이러한 결과 는 아동이 학교 현장에서 성공적으로 적응하는 것을 돕기 위 한 중재 프로그램을 설계하는 데 활용될 수 있으며, 특히 이러 한 프로그램에 주의를 통제하고 유연한 사고를 증진하도록 하 는 훈련이 포함될 필요가 있음을 강조한다. 또한 아동의 성공 적인 학교적응을 위해서는 가정과 학교에서 아동이 자신의 능 력을 최대한 발달시키도록 돕는 것뿐만 아니라, 스스로의 능 력에 대한 믿음을 키워줄 수 있도록 아동에게 다양한 성취 경 험을 해볼 수 있는 환경을 제공해 주는 것이 중요하다는 것을 시사한다.

마지막으로 본 연구의 제한점과 후속연구를 위한 제언을 해보면 다음과 같다. 첫째, 본 연구에서는 학령기 아동의 실행 기능을 측정하는 데 있어 실행기능의 하위요인 중 대표적인 억제 능력, 작업 기억 및 전환 능력만을 포함하였다는 제한점 이 있다. 비록 이러한 능력들이 실행기능을 대표하기는 하지 만, 실행기능이 고차원적인 인지기능의 집합체라는 점을 고려 해 볼 때, 보다 많은 과제를 통해 학령기 아동의 실행기능을 통 합적으로 파악할 필요가 있다. 따라서 후속 연구에서는 학령 기 아동에게 필요한 계획 및 조직화와 관련된 실행기능 과제 
들을 함께 실시함으로써 실행기능을 다양한 측면에서 측정할 수 있도록 하는 시도가 필요할 것이다. 둘째, 본 연구는 과제 수행을 통해 실행기능을 측정하였는데, 과제로 측정하는 실행 기능은 아동이 일상생활에서 보이는 실행기능을 적절하게 반 영하지 못할 가능성, 즉 생태학적 타당성이 낮을 가능성이 있 다. 이러한 측면에서 실행기능을 질문지로 측정하는 도구들 (Gioia, Espy, \& Isquith, 2003; Song, 2014)이 개발되어 사용되 고 있다. 따라서 후속 연구에서는 과제 수행과 실행기능 척도 를 함께 사용해서 아동의 실행기능을 측정한다면 실행기능을 보다 통합적으로 측정하는 데 도움이 될 것으로 기대된다. 마 지막으로, 본 연구에서는 자기효능감이 학교적응에 선행하는 것으로 모형을 설정하였지만, 연구 시점 이전의 아동의 학교 적응에 대한 경험이 아동의 자기효능감에 영향을 미쳤을 수도 있다. 따라서 후속 연구에서는 종단 연구 설계를 통해 아동의 자기효능감이 학교적응에 미치는 인과관계를 더욱 면밀하게 확인해 볼 필요성이 있다.

이러한 제한점에도 불구하고 본 연구는 다음과 같은 몇 가 지 의의를 지닌다. 먼저, 지금까지의 실행기능 연구는 주로 영 유아기를 대상으로 수행되어왔다. 학령기는 실행기능의 급격 한 발달 시기를 지나고 상대적으로 안정기에 접어드는 시기이 지만, 이 시기 실행기능이 지속적으로 향상되고 있는 것은 분 명하다(Huizinga, Dolan, \& van der Molen, 2006). 따라서 학령 기 아동의 실행기능에 관한 연구도 계속적으로 축적될 필요성 이 있으며, 이 시기 실행기능이 다양한 발달 결과에 미치는 영 향력도 탐색해볼 필요가 있다. 이러한 점에서 본 연구는 학령 기 아동을 대상으로 과제를 통해 실행기능을 측정하였으며, 이 시기 실행기능이 아동의 학교에서의 적응에 미치는 영향력 을 파악하였다는 데 의의가 있다. 또한 지금까지의 연구들은 아동이 실제로 가지고 있는 능력인 실행기능과 자신의 능력에 대한 믿음인 자기효능감을 함께 살펴보지 않았다. 그러나 본 연구에서 학령 후기 아동의 학교적응에 영향을 미치는 실제 능력인 실행기능과 능력에 대한 믿음인 자기효능감으로 구분 하여 살펴보았다는 데 의의가 있다. 이러한 결과는 아동의 실 제 능력을 증진 시켜주는 것도 중요하지만, 아동이 스스로 자 신의 능력을 믿을 수 있도록 돕기 위한 개입이 필요함을 제시 한다. 본 연구의 결과는 교육 및 상담 현장에서 다양한 학교적 응 증진 프로그램을 개발할 때 기초자료로 활용될 수 있을 것 으로 기대한다.

\section{Acknowledgements}

This work was supported by the Ministry of Education of the Republic of Korea and the National Research Foundation of Korea (NRF-2016S1A3A2924375)

\section{Notes}

This article is a part of the first author's master thesis submitted in 2019, and was presented at the 2019 Annual Spring Conference of the Korean Association of Child Studies.

\section{Conflict of Interest}

No potential conflict of interest relevant to this article was reported.

\section{References}

\section{In English}

Anderson, P. (2002). Assessment and development of Executive Function (EF) during childhood. Child Neuropsychology, 8(2), 71-82. doi:10.1076/chin.8.2.71.8724

Aunola, K., Stattin, H., \& Nurmi, J.-E. (2000). Adolescents' achievement strategies, school adjustment, and externalizing and internalizing problem behaviors. Journal of Youth and Adolescence, 29(3), 289-306. doi:10.1023/A:1005143607919

Baddeley, A. (2003). Working memory: Looking back and looking forward. Nature Reviews Neuroscience, 4, 829-839. doi:10.1038/nrn1201

Bandura, A. (1977). Self-efficacy: Toward a unifying theory of behavioral change. Psychological Review, 84(2), 191-215. doi:10.1037/0033-295X.84.2.191

Bandura, A. (1982). Self-efficacy mechanism in human agency. American Psychologist, 37(2), 122-147. doi:10.1037/0003066X.37.2.122

Bandura, A. (1993). Perceived self-efficacy in cognitive development and functioning. Educational Psychologist, 28(2), 117-148. doi:10.1207/s15326985ep2802_3

Bandura, A., \& Cervone, D. (1983). Self-evaluative and selfefficacy mechanisms governing the motivational effects of goal systems. Journal of Personality and Social Psychology, 45(5), 1017-1028. doi:10.1037/0022-3514.45.5.1017

Baron, R. M., \& Kenny, D. A. (1986). The moderator-mediator 
variable distinction in social psychological research: Conceptual, strategic, and statistical considerations. Journal of Personality and Social Psychology, 51(6), 1173-1182.

Beveridge, M., Jarrold, C., \& Pettit, E. (2002). An experimental approach to executive fingerprinting in young children. Infant and Child Development, 11(2), 107-123. doi:10. 1002/icd.300

Birch, S. H., \& Ladd, G. W. (1997). The teacher-child relationship and children's early school adjustment. Journal of School Psychology, 35(1), 61-79.

Carlson, S. M. (2005). Developmentally sensitive measures of executive function in preschool children. Developmental Neuropsychology, 28(2), 595-616. doi:10.1207/s15326 942dn2802_3

Cepeda, N. J., Kramer, A. F., \& Gonzalez de Sather, J. C. M. (2001). Changes in executive control across the life span: Examination of task-switching performance. Developmental Psychology, 37(5), 715-730. doi:10.1037/00121649.37.5.715

Coleman, P. K. (2003). Perceptions of parent-child attachment, social self-efficacy, and peer relationships in middle childhood. Infant and Child Development, 12(4), 351-368. doi:10.1002/icd.316

Daneman, M., \& Carpenter, P. A. (1980). Individual differences in working memory and reading. Journal of Verbal Learning and Verbal Behavior, 19(4), 450-466. doi:10.1016/S00225371(80)90312-6

Diamond, A. (2013). Executive functions. Annual Review of Psychology, 64, 135-168. doi:10.1146/annurev-psych113011-143750

DuBois, D. L., Eitel, S. K., \& Felner, R. D. (1994). Effects of family environment and parent-child relationships on school adjustment during the transition to early adolescence. Journal of Marriage and Family, 56(2), 405414. doi: $10.2307 / 353108$

Engle, R. W. (2002). Working memory capacity as executive attention. Current Directions in Psychological Science, 11(1), 19-23. doi:10.1111/1467-8721.00160

Gambin, M., \& Święcicka, M. (2015). Relationships of self-efficacy beliefs to executive functions, hyperactivity-impulsivity and inattention in school-aged children. Polish Journal of Applied Psychology, 13(1), 33-42. doi:10.1515/pjap-2015-0024

Gathercole, S. E., Lamont, E., \& Alloway, T. P. (2006). Working memory in the classroom. In S. J. Pickering (Ed.), Working memory and education (pp. 219-240). Burlington, MA: Academic Press.

Gioia, G. A., Espy, K. A., \& Isquith, P. K. (2003). BRIEF-P: Behavior Rating Inventory of Executive Function-Preschool version. Odessa, FL: Psychological Assessment Resources.

Harter, S. (1982). The perceived competence scale for children.
Child Development, 53(1), 87-97. doi:10.2307/1129640

Heaton, R. K., Chelune, G. J., Talley, J. L., Kay, G. G., \& Curtiss, G. (1993). Wisconsin card sorting test manual: Revised and expanded (2nd ed.). Odessa, FL: Psychological Assessment Resources.

Huizinga, M., Dolan, C. V., \& van der Molen, M. W. (2006). Agerelated change in executive function: Developmental trends and a latent variable analysis. Neuropsychologia, 44(11), 2017-2036. doi:10.1016/j.neuropsychologia.2006.01.010

Jacobson, L. A., Williford, A. P., \& Pianta, R. C. (2011). The role of executive function in children's competent adjustment to middle school. Child Neuropsychology, 17(3), 255-280. doi: 10.1080/09297049.2010.535654

Ladd, G. W., Kochenderfer, B. J., \& Coleman, C. C. (1997). Classroom peer acceptance, friendship, and victimization: Distinct relational systems that contribute uniquely to children's school adjustment? Child Development, 68(6), 1181-1197. doi:10.1111/j.1467-8624.1997.tb01993.x

LeFevre, J.-A., Berrigan, L., Vendetti, C., Kamawar, D., Bisanz, J., Skwarchuk, S.-L., \& Smith-Chant, B. L. (2013). The role of executive attention in the acquisition of mathematical skills for children in grades 2 through 4 . Journal of Experimental Child Psychology, 114(2), 243-261. doi:10.1016/ j.jecp.2012.10.005

Liem, A. D., Lau, S., \& Nie, Y. (2008). The role of self-efficacy, task value, and achievement goals in predicting learning strategies, task disengagement, peer relationship, and achievement outcome. Contemporary Educational Psychology, 33(4), 486-512. doi:10.1016/j.cedpsych.2007.08.001

Liew, J., McTigue, E. M., Barrois, L., \& Hughes, J. N. (2008). Adaptive and effortful control and academic self-efficacy beliefs on achievement: A longitudinal study of 1 st through 3rd graders. Early Childhood Research Quarterly, 23(4), 515526. doi:10.1016/j.ecresq.2008.07.003

Luna, B., \& Sweeney, J. A. (2004). The emergence of collaborative brain function: fMRI studies of the development of response inhibition. Annals of the New York Academy of Sciences, 1021(1), 296-309. doi:10.1196/annals.1308.035

Lynch, M., \& Cicchetti, D. (1997). Children's relationships with adults and peers: An examination of elementary and junior high school students. Journal of School Psychology, 35(1), 81-99. doi:10.1016/S0022-4405(96)00031-3

Monette, S., Bigras, M., \& Guay, M.-C. (2011). The role of the executive functions in school achievement at the end of grade 1. Journal of Experimental Child Psychology, 109(2), 158-173. doi:10.1016/j.jecp.2011.01.008

Pessoa, L. (2009). How do emotion and motivation direct executive control? Trends in Cognitive Sciences, 13(4), 160-166. doi:10.1016/j.tics.2009.01.006

Riggs, N. R., Blair, C. B., \& Greenberg, M. T. (2004). Concurrent 
and 2-year longitudinal relations between executive function and the behavior of 1st and 2nd grade children. Child Neuropsychology, 9(4), 267-276. doi:10.1076/ chin.9.4.267.23513

Rothbart, M. K., Sheese, B. E., \& Posner, M. I. (2007). Executive attention and effortful control: Linking temperament, brain networks, and genes. Child Development Perspectives, 1(1), 2-7. doi:10.1111/j.1750-8606.2007.00002.x

Rubin, K. H., Bukowski, W., \& Parker, J. (2006). Peers, relationships, and interactions. In W. Damon \& R. M. Lerner (Series Eds.) \& N. Eisenberg (Vol. Ed.), Handbook of child psychology: Volume 3. social, emotional, and personality development (6th ed., pp. 571-645). Hoboken, NJ: John Willey \& Sons.

Sasser, T. R., Bierman, K. L., \& Heinrichs, B. (2015). Executive functioning and school adjustment: The mediational role of pre-kindergarten learning-related behaviors. Early Childhood Research Quarterly, 30, 70-79. doi:10.1016/ j.ecresq.2014.09.001

Schunk, D. H. (1989). Self-efficacy and achievement behaviors. Educational Psychology Review, 1(3), 173-208.

Scott, W. A. (1962). Cognitive complexity and cognitive flexibility. Sociometry, 25(4), 405-414.

Valiente, C., Lemery-Chalfant, K., Swanson, J., \& Reiser, M. (2008). Prediction of children's academic competence from their effortful control, relationships, and classroom participation. Journal of Educational Psychology, 100(1), 6777. doi:10.1037/0022-0663.100.1.67

Visu-Petra, L., Cheie, L., Benga, O., \& Miclea, M. (2011). Cognitive control goes to school: The impact of executive functions on academic performance. Procedia-Social and Behavioral Sciences, 11, 240-244. doi:10.1016/j.sbspro.2011.01.069

Welsh, M. C. (2002). Developmental and clinical variations in executive functions. In D. L. Molfese \& V. J. Molfese (Eds.), Developmental variations in learning: Applications to social, executive function, language, and reading skills (pp. 139185). Mahwah, NJ: Lawrence Erlbaum Associates.

Williams, B. R., Ponesse, J. S., Schachar, R. J., Logan, G. D., \& Tannock, R. (1999). Development of inhibitory control across the life span. Developmental Psychology, 35(1), 205213.

Zelazo, P. D., \& Müller, U. (2002). Executive function in typical and atypical development. In U. Goswami (Ed.), Blackwell handbook of childhood cognitive development (pp. 445-469). Malden, MA: Blackwell Publishers. doi:10.1002/9780470996652.ch20

Zelazo, P. D., Müller, U., Frye, D., Marcovitch, S., Argitis, G., Boseovski, J., . . . \& Carlson, S. M. (2003). The development of executive function in early childhood. Monographs of the Society for Research in Child Development,
68(3), 1-151. doi:10.1111/j.0037-976X.2003.00261.x

Zorza, J. P., Marino, J., \& Mesas, A. A. (2016). Executive functions as predictors of school performance and social relationships: Primary and secondary school students. The Spanish Journal of Psychology, 19(e23), 1-10. doi:10.1017/sjp.2016.23

\section{In Korean}

Cho, S. (2014). Development and validation of the school adjustment scale for school-aged children (Doctoral dissertation). Retrieved from http://www.riss.kr/link?id=T13522341

Cho, Y.-J. (2005). Relationships among sport participation, selfefficacy and school adjustment in elementary school students. Journal of Korean Society for The Study of Physical Education, 10(1), 109-121.

Cho, Y. S., \& Lee, K. N. (2010). Effects of adolescent's alienation, depression, family environment and school maladjustment on suicidal ideation. Journal of the Korean Home Economics Association, 48(8), 27-37.

Choi, Y. J., \& Lee, S. Y. (2016). The relationship between early adolescent's parent attachment and quality of peer relationship: Mediating effect of executive function and adaptive cognitive emotion regulation. The Korean Journal of Applied Developmental Psychology, 5(1), 53-73.

Chung, Y. L., \& Kim, S. O. (2012). Analysis of metacognition, self-efficacy, and perceptions of the constructivist learning environment in science for middle and high school students. Journal of Research in Curriculum Instruction, 16(1), 125-144.

Do, K. M. (2015). The mediating effects of intimacy and cognitive flexibility in effects of humor style on school adaptation (Master's thesis). Retrieved from http://www.riss.kr/ link?id=T13642123

Han, H. J. (2002). The effects of self assertiveness training on the development of children's self-efficacy (Master's thesis). Retrieved from http://www.riss.kr/link?id=T8154303

Jang, S.-H., Choi, E.-H., \& Lee, J.-Y. (2016). The mediating effect of ego-resilience in the effect of elementary student's psychological well-being on adjustment to school life and everyday life stress. The Journal of Child Education, 25(2), 353-364. doi:10.17643/KJCE.2016.25.2.16

Jin, A. (2018). Effects of working memory and text types (narrative and expository text) on Korean elementary school students' english learning (Master's thesis). Retrieved from http:// www.riss.kr/link?id=T14719200

Ju, D.-B. (2011). The relationships among the elementary school student's self-control, self-efficacy, and school life adjustment: Focusing on Busan area. Journal of Fisheries and Marine Sciences Education, 23(2), 277-289.

Jung, M. (2008). The relationship between the communication 
levels of class teachers'-children perceived by elementary school students and adjustment to school life-The role of self-efficacy as a mediator (Master's thesis). Retrieved from http://www. riss.kr/link?id=T11437392

Kang, I., \& Kim, C. (2014). A structural analysis of emotional intelligence, self-efficacy, and self-directed learning attitudes perceived by elementary school students. Journal of LearnerCentered Curriculum and Instruction, 14(12), 115-137.

Kim, H., \& Park, T. (2003). Korean norm for the difference between digits forward and digits backward. The Korean Journal of Clinical Psychology, 22(3), 599-613.

Kim, H.-S. (2019). The effect of perceived social support and academic self-efficacy of innovative elementary school students on school happiness (Master's thesis). Retrieved from http://www.riss. kr/link?id=T15099331

Kim, M., \& Ha, E. H. (2013). The mediating effects of executive functions on the relationships between adolescent's ADHD symptoms and quality of peer relationships. Korean Journal of Clinical Psychology, 32(2), 313-330.

Kim, M. S. (2009). The study of the effect of perceived stress and depression on the school adjustment of Korean adolescents. The Journal of the Korean Association of Psychology, 1(2), 5367.

Kwak, K., Oh, S., \& Kim, C. (2011). Korean-Wechsler Intelligence Scale for Children-Forth edition (K-WISC$I V)$ : Administration and scoring manual. Seoul: Insight of Psychology.

Lee, H. N., \& Lee, S. (2012). The path analysis of emotional support, emotion regulation and self-efficiency affecting school adjustment of children. Journal of the Korean Home Economics Association, 50(2), 65-74. doi:10.6115/ khea.2012.50.2.065

Lee, J.-I., Kim, C.-K., \& Han, E.-S. (2014). A structural analysis of parental attachment, peer attachment, self-efficacy, and school adjustment perceived by elementary school students. The Journal of Elementary Education, 27(4), 345-367.

Lee, M.-J., \& Hong, C.-H. (2006). Dimensions and development of executive function. The Korean Journal of Clinical Psychology, 25(2), 587-602.

Lee, M.-S., \& Lee, G.-M. (2009). A study on academic achievement, self-concept, school-related adjustment, and school maladjustment behavior of vocational high school students. The Journal of Educational Studies, 40(1), 167193.
Lee, Y., \& Kim, D. (2018). Mediating effects of executive function deficits in adolescent's ADHD tendency, self-competence, and school life adjustment. Asian Journal of Education, 19(3), 703-723. doi:10.15753/aje.2018.09.19.3.703

Min, Y. (1994). Gyoyuksimnihak [교육심리학]. Yongin, KR: Moonumsa.

Ministry of Education. (2018). 2018nyeon gyoyukgibontonggye juyonaeyong [2018년 교육기본통계 주요내용]. Retrieved from MOE website: https://www.moe.go.kr

Park, H.-L., \& Park, S.-O. (2016). Effect of children's maladjustment to school on smartphone addiction: Mediating effect of depression and aggression. Korean Journal of Infant Mental Health, 9(2), 53-79.

Shin, M., \& Koo, H. (2006). A standardization study for Korean version of Children's Color Trails Test (CCTT). In The Korean Psychological Association. (Ed.), Proceedings of 2006 Conferences of the Korean Psychological Association (pp. 648-649). Seoul: Auhtor.

Shin, M., \& Park, M. (2006). A standardization study for Korean version of stroop color-word test children's version. In The Korean Psychological Association. (Ed.), Proceedings of 2006 Conferences of the Korean Psychological Association (pp. 628-629). Seoul: Auhtor.

Shin, Y. H., Yun, J. Y., \& Lee, S. B. (2010). A study on executive function and problem behavior type for a children with maladjustment behaviors in school environment and general children. Journal of Special Education \& Rehabilitation Science, 49(1), 197-213.

Song, H. (2014). Validity of child-adolescent self-reported executive function difficulty screening questionnaire. Korean Journal of Clinical Psychology, 33(1), 121-137.

\section{ORCID}

Jae Eun Jeong http://orcid.org/0000-0001-5850-5659

Nana Shin http://orcid.org/0000-0002-7869-6875

Received June 30, 2019

Revision received July 20, 2019

Accepted July 23, 2019 